\title{
INFLUENCE DE L'ABROUTISSEMENT PAR LE LAPIN DE GARENNE D'UNE CULTURE DE BLE D'HIVER
}

\author{
C.T.G.R.E.F.-Nogent sur Vermisson \\ Compte rendu d'expérience $1977 / 2$
}

Class. Oxford $156.5: 149.32:$ CDU 632.6

Résumé : La comparaison des hauteurs et rendements de blé d'hiver sur des zones protégées et non protégées a montré que l'influence du lapin sur la croissance de la céréale jusqu'à l'épiaison était importante, mais que la pression d'abroutissement s'exerçant principalement dans les dix premiers mètres en bordure de la culture affecte peu le rendement global.

\section{BUT DE L'EXPÉRIMENTATION}

Les détériorations de certaines cultures agricoles et principalement des céréales d'hiver par le lapin de garenne pendant la période hivernale peuvent être estimées visuellement en pourcentage de la surface totale concernée mais seule la comparaison des croissances et des rendements dans des placettes protégées et non protégées permet de chiffrer les pertes et d'obtenir ainsi une estimation objective des dommages.

Les observations et mesures effectuées dans une culture de blé d'hiver au cours de son cycle végétatif avaient un triple but :

- évaluer la perte en matière verte et le retard de croissance subis par la céréale;

- estimer la perte en grain à la moisson;

- déterminer le moment le plus favorable à l'installation d'un dispositif de protection efficace, lorsque celui-ci est jugé nécessaire.

\section{DESCRIPTION DE L'EXPÉRIENCE}

L'expérience a été réalisée sur le Domaine des Barres (Loiret) au cours des années agricoles 1974-75 et 1975-76 chaque fois dans un site différent, mais de même type de sol (rendzine sur calcaire de Beauce) et supportant un effectif de lapins assez voisin (10 animaux/ha environ).

Dès le semis, trois enclos grillagés (maille $36 \mathrm{~mm}$ ) de $1,10 \mathrm{~m}$ de hauteur et de $3 \mathrm{~m} \times 25 \mathrm{~m}$ (expérimentation $A-1974-75$ ) et $2,5 \mathrm{~m} \times 20 \mathrm{~m}$ (expérimentation $\mathrm{B}-1975-76$ ) furent mis en place et disposés en bordure de champ, à proximité des zones de provenance des lapins. 


\section{EXPÉRIMENTATION A (1974-75)}

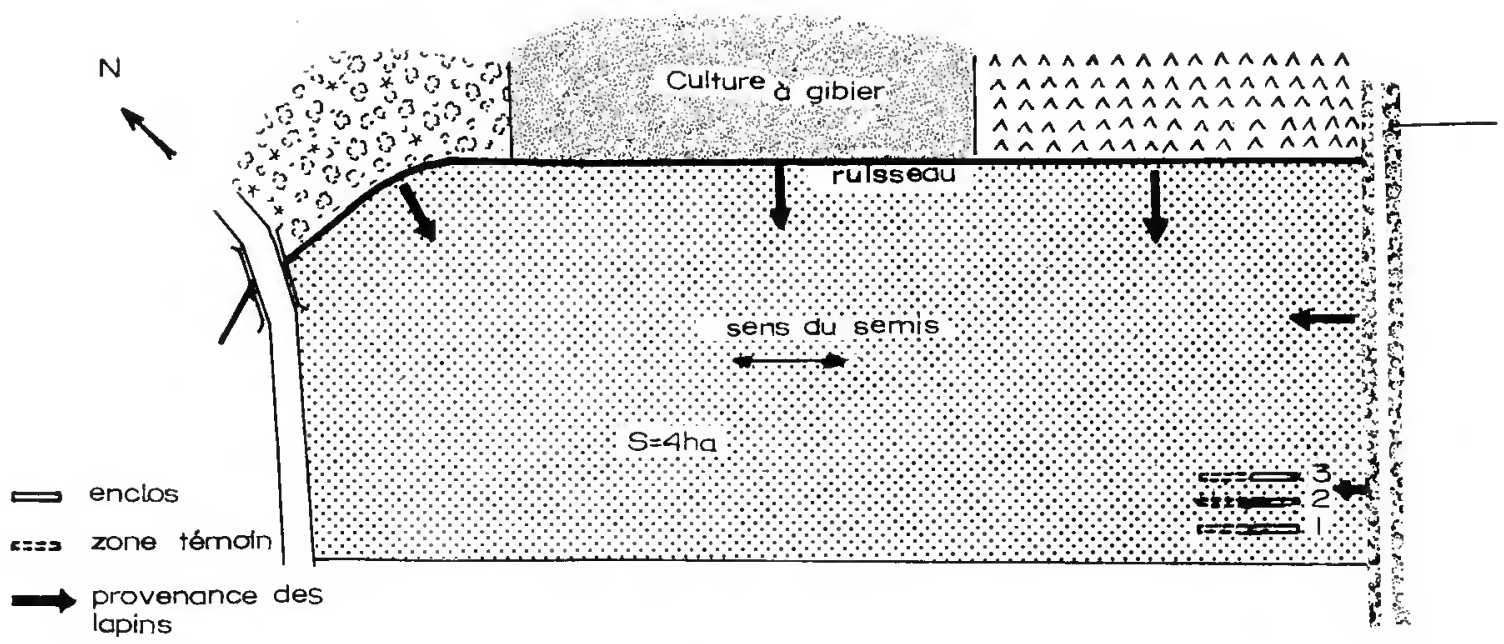

\section{EXPÉRIMENTATION B (1975-76)}

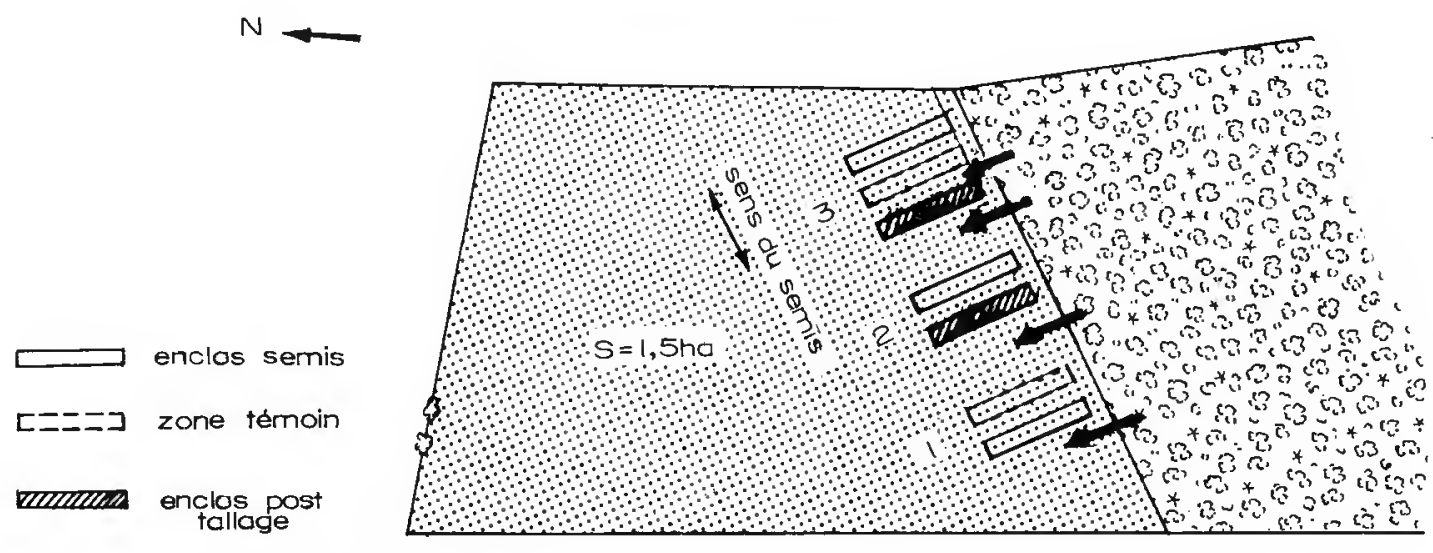

$\rightarrow$ provenance des lapins

Dans l'expérimentation $B$, deux enclos supplémentaires, situés entre ceux déjà présents furent installés à l'époque du tallage (enclos post-tallage) vers la mi-mars 1976.

La situation des enclos et des zones témoins, utilisés pour les divers prélèvements, figure sur les schémas ci-dessus.

Certains enclos n'ont été utilisés pour les mesures qu'à certaines périodes de végétation et les zones parcourues et piétinées fréquemment lors des premières mesures ont dû être abandonnées pour les prélèvements d'échantillons en fin d'expérimentation.

Les observations et dénombrements (comptage de semis, mesure de la hauteur des feuilles et épis, mesure de la productivité) ont été effectués sur 50 à 100 placettes de $0,1 \mathrm{~m}^{2}$ à l'intérieur et à l'extérieur des enclos. 
Expérimentation A. Vue d'ensemble du dispositif début avril 1975.

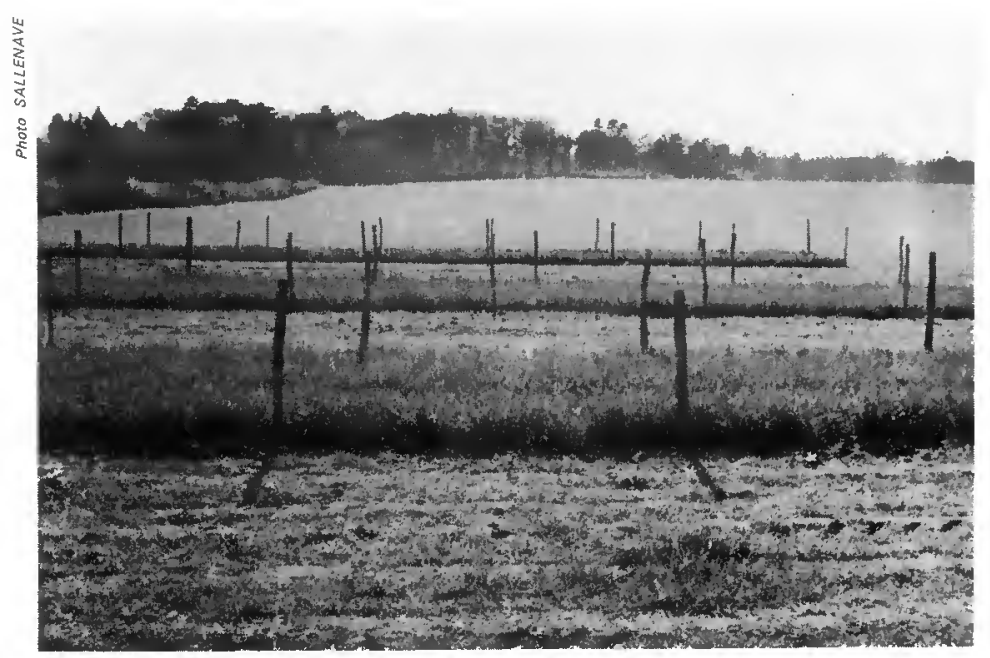

Expérimentation B. Vue d'un placeau début avril 1976 montrant la situation par rapport au bois.

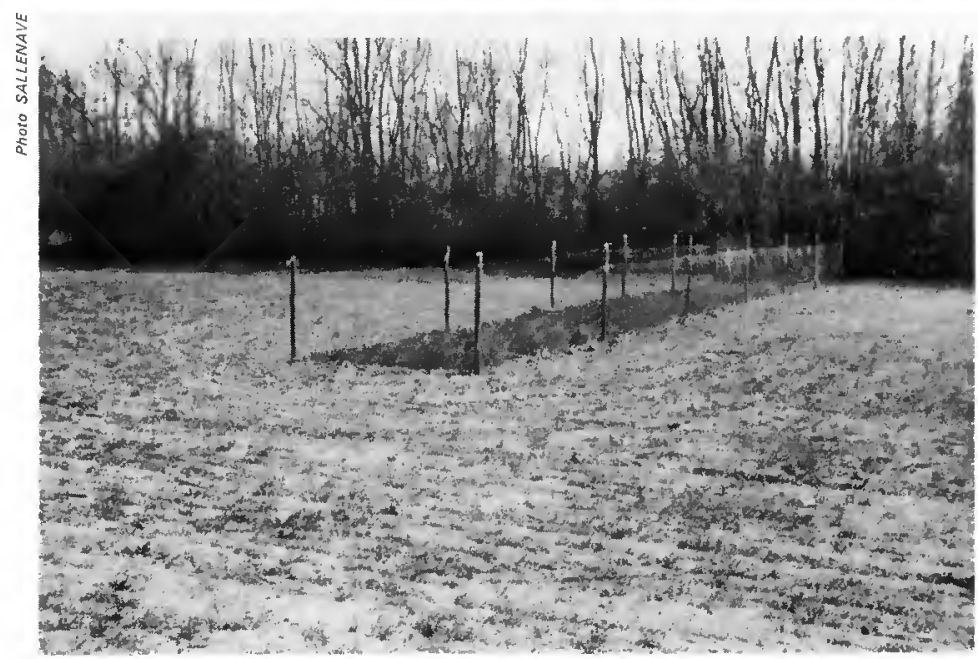

Le calendrier des opérations a été le suivant :

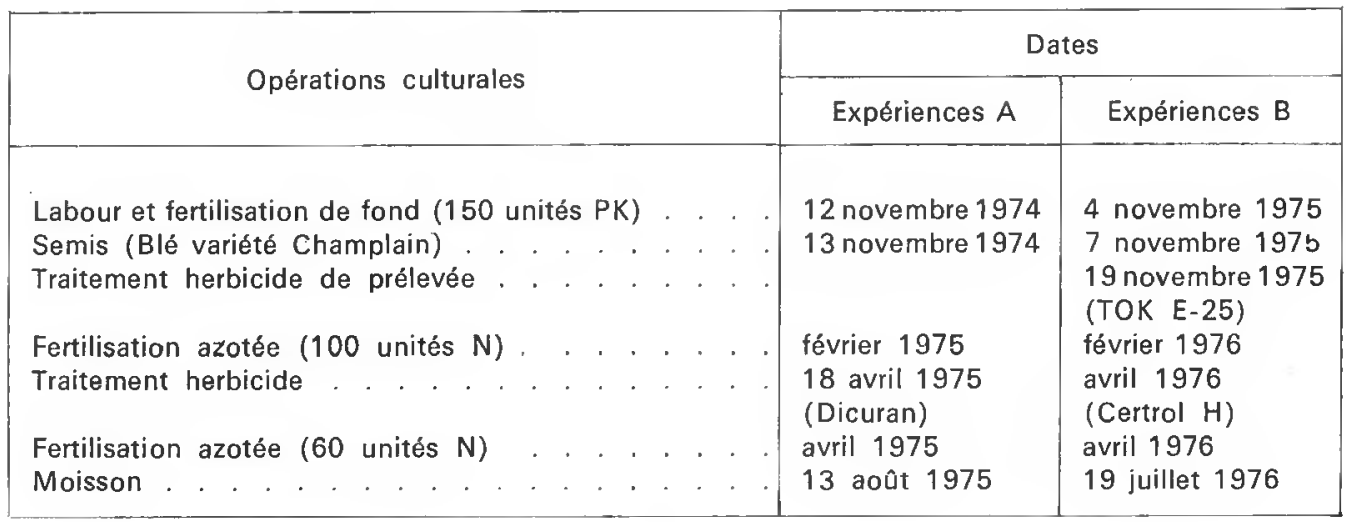




\section{RÉSULTATS}

\section{Influence sur la densité du semis et la formation des racines}

Les densités de semis à l'intérieur et à l'extérieur des enclos sont identiques, de l'ordre de 250 pieds au $\mathrm{m}^{2}$ dans l'expérimentation $A$ et de 310 pieds dans l'expérimentation $B$, ce qui confirme le fait que le lapin tond, mais n'arrache pas la plante.

On a noté quelques différences dans le nombre de racines secondaires formées sur les plants situés à l'extérieur et à l'intérieur des enclos : ainsi $76 \%$ des plants protégés ont 5 racines et plus contre $45 \%$ seulement des plants non protégés.

\section{Influence sur la morphologie au cours du cycle végétatif}

Les o'bservations sur la croissance effectuées jusqu'à l'épiaison dans l'expérimentation $A$ et jusqu'au tallage pour l'expérimentation B, sont résumées dans les tableaux ci-après.

\section{Expérimentation A}

\begin{tabular}{|c|c|c|c|c|c|c|}
\hline & \multicolumn{4}{|c|}{$\begin{array}{l}\text { Longueur de la plus grande feuille } \\
\qquad(\mathrm{mm})\end{array}$} & \multicolumn{2}{|c|}{ Hauteur épi (mm) } \\
\hline & $15-01-75$ & $4-02-75$ & $25-02-75$ & $25-03-75$ & $4-06-75\left(^{*}\right)$ & $30-06-75\left({ }^{* *}\right)$ \\
\hline Enclos . . . . . & $96 \pm 5,5$ & $110 \pm 6$ & $133 \pm 6$ & $162 \pm 6$ & $980 \pm 14$ & $1023 \pm 16$ \\
\hline Zone témoin . . & $25 \pm 3$ & $22 \pm 2$ & $36 \pm 7$ & $35 \pm 4$ & $699 \pm 24$ & $857 \pm 20$ \\
\hline Milieu du champ & & $22 \pm 3$ & $22 \pm 4$ & $38 \pm 5$ & & \\
\hline
\end{tabular}

\section{Expérimentation $B$}

\begin{tabular}{|c|c|c|c|}
\hline & \multicolumn{3}{|c|}{ Longueur de la plus grande feuille (mm) } \\
\hline & $19-01-67$ & $17-02-76$ & $26-03-76$ \\
\hline Enclos 1 . & $72 \pm 3$ & $87 \pm 2$ & $147 \pm 4$ \\
\hline Zone témoin $1 \ldots \ldots$ & $41 \pm 3$ & $46 \pm 3$ & $79 \pm 5$ \\
\hline 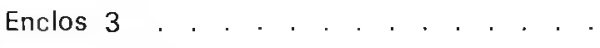 & $74 \pm 3$ & $98 \pm 3$ & $155 \pm 4$ \\
\hline Zone témoin $3 \ldots \ldots$ & $61 \pm 3$ & $49 \pm 3$ & $80 \pm 6$ \\
\hline Enclos post-tallage & & & $92 \pm 9$ \\
\hline Milieu du champ. . . . . . . . & & 51,9 & 94,4 \\
\hline
\end{tabular}

Les mensurations qui ont débuté en janvier deux mois après le semis, font apparaître une différence très sensible des hauteurs de blé entre les zones protégées et non protégées : 
Vue prise le 12 juin 1975 , mettant en évidence le dècalage de l'épiaison entre I'enclos (à gauche) et l'extérieur.

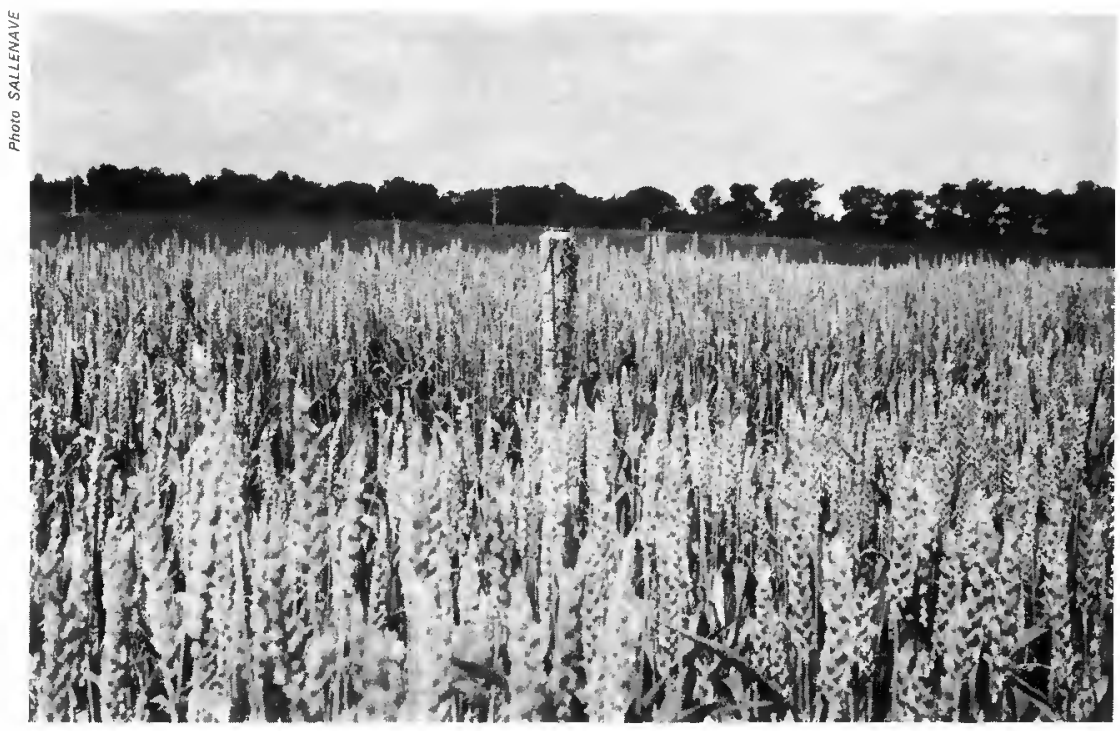

ainsi la hauteur de la céréale est réduite avant épiaison de 73 à $80 \%$ dans l'expérimentation $A$ et de 20 à $50 \%$ dans l'expérimentation B.

Le prélèvement effectué par le lapin, de l'époque du semis à février, a été évalué à $250 \mathrm{~kg} / \mathrm{ha}$ de matière verte, soit environ $4.3 \mathrm{~kg} / \mathrm{ha} /$ jour pour une densité de 10 lapins à l'hectare (expérimentation $\mathrm{A}$ ).

Dans'l'expérimentation A, la pression du lapin sur les zones témoins s'exerce de façon pratiquement uniforme, ce qui s'explique par la disposition des placeaux. En revanche dans l'expérimentation B, les prélèvements varient en fonction de l'éloignement de la zone boisée. On peut ainsi constater, à la lecture des graphiques 1 et 2, que peu de temps après le tallage (notation du 26 mars), il existe des différences très nettes entre les hauteurs sur les zones témoins en fonction de l'éloignement de la bordure, alors qu'à l'intérieur des enclos, ces différences sont minimes (ce qui montre d'ailleurs qu'à ce stade de végétation, il n'y a aucun " effet de bordure").

A maturation (avant moisson), un comptage du nombre de tiges portant des épis sur une cinquantaine de placeaux de $0,1 \mathrm{~m}^{2}$ a donné les moyennes suivantes :

\begin{tabular}{|l|c|c|c|}
\hline \multirow{2}{*}{} & \multicolumn{3}{|c|}{ Nombre de tiges sur $0,1 \mathrm{~m}^{2}$} \\
\cline { 2 - 4 } & Enclos & Témoin & $\begin{array}{c}\text { Enclos } \\
\text { post-tallage }\end{array}$ \\
\hline Expérimentation A & $43 \pm 3$ & $46 \pm 3$ & \\
\hline Expérimentation B & 48,6 & 39,8 & 44,7 \\
\hline
\end{tabular}

Les résultats chiffrés des mesures faites à diverses distances de la bordure (expérimentation B) sont reportés sur le graphique 3. Celui-ci montre :

- qu'il existe une différence importante du nombre d'épis formés dans les enclos et les zones témoins, valeur assez constante dans les 10 premiers mètres: 
- que le nombre d'épis augmente notablement depuis la bordure jusqu'à 10-14 m, tant dans les zones protégées que dans les zones témoins

- qu'au-delà de cette distance (10-14 m), les effets «lapin » et «bordure » ne se manifestent plus que faiblement.

\section{Influence sur le rendement}

Les rendements moyens obtenus hors et en enclos ont été les suivants :

\begin{tabular}{|c|c|}
\hline Expérimentation A & $\begin{array}{l}\text { Rendement } \\
\text { (q/ha) }\end{array}$ \\
\hline Enclos $1 \ldots .$. & 35,2 \\
\hline Enclos $2 \ldots . .$. & 38,6 \\
\hline Témoin. . & 38,8 \\
\hline Ensemble du champ. & 35,0 \\
\hline
\end{tabular}

\begin{tabular}{|l|c|}
\hline Expérimentation B & $\begin{array}{c}\text { Rendement } \\
(\mathrm{q} / \mathrm{ha})\end{array}$ \\
\hline Enclos semis . . . . & 32,6 \\
\hline Enclos post-tallage . . . & 33,0 \\
\hline Témoin . . . . . . & 31,1 \\
\hline Ensemble du champ . . . & 35,0 \\
\hline
\end{tabular}

Les rendements hors et en enclos sont voisins dans les deux cas; il semble bien que l'abroutissement intensif du lapin au cours de l'hiver n'ait eu qu'une très faible influence sur le rendement moyen final et que, dans le cas qui nous préoccupe, le gain que l'on peut espérer par la mise en place d'un engrillagement soit trop faible pour justifier une telle opération.

Cependant, dans l'expérimentation $B$, le rendement varie, comme le nombre d'épis formés, en fonction de l'éloignement des bordures comme le montrent les graphiques nos 3 et 4 et le tableau ci-après (expérimentation B).

\begin{tabular}{|c|c|c|c|}
\hline \multirow{2}{*}{ Distance de la bordure } & \multicolumn{3}{|c|}{ Rendements (q/ha) } \\
\cline { 2 - 4 } & $\begin{array}{c}\text { Enclos } \\
\text { semis }\end{array}$ & $\begin{array}{c}\text { Enclos } \\
\text { post-tallage }\end{array}$ & $\begin{array}{c}\text { Zone } \\
\text { témoin }\end{array}$ \\
\hline $0-10 \mathrm{~m}$ & 25,2 & 22,7 & 19,6 \\
\hline $10-22 \mathrm{~m}$ & 40,0 & 43,3 & 42,6 \\
\hline
\end{tabular}

Ce sont les dix premiers mètres, intensivement abroutis par le lapin au cours de l'hiver qui accusent la perte de rendement la plus élevée (5,6 q/ha par rapport à la même zone protégée), la proximité du bois exerçant dans tous les cas une influence négative prépondérante sur la productivité.

\section{CONCLUSION}

Dans les conditions particulières de l'expérimentation (dénsité importante de lapins, blé à fort pouvoir de tallage semé assez serré) il s'avère que, si l’action du lapin nuit considérablement à la croissance de la céréale, les effets de l'abroutissement n'affectent que peu la production et seulement sur une zone où elle est déjà amoindrie par la concurrence du boisement. 
Toute généralisation des observations faites pendant deux saisons agricoles seulement serait certes hasardeuse et l'expérimentation sera poursuivie avec d'autres types de cultures d'hiver ou de printemps (autres variétés de blé, maïs, colza ...). Mais il apparaît d'ores et déjà qu'il convient de se garder de toute appréciation hâtive sur la réussite d'un emblavement soumis à la dent du lapin et de toute décision qui pourrait en être la conséquence. Ce n'est qu'au vu de la production que l'on pourra apprécier l'importance des dommages.

\section{ANNEXE}

L'expérimentation a été reprise en 1976/77 sur le blé d'hiver (variété Talent) et sur orge de printemps. Des différences de rendement significatives ont été observées cette fois dans les deux cas.

Pour le blé d'hiver, la perte est de l'ordre de $12 \mathrm{qx} /$ ha sur une profondeur que l'on peut évaluer à $20 / 25 \mathrm{~m}$ (la différence s'atténuant tout en demeurant significative sur les placeaux situés entre 16 et $20 \mathrm{~m}$ ).

Pour l'orge, la différence est plus importante au départ (production quasiment nulle sur la bande $0.4 \mathrm{~m}$ ), mais s'atténue plus rapidement pour devenir non significative à partir de $12 \mathrm{~m}$. II reste que les dégâts de lapins ne portent que sur une zone limitée, zone également influencée par les effets de bordure.

Graphique no 1

\section{ÉVOLUTION DANS LE TEMPS DE LA HAUTEUR DU BLÉ EN FONCTION DE L'ÉLOIGNEMENT DE LA BORDURE (Expérience B)}

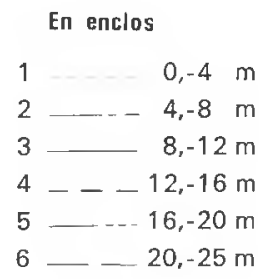

Zone témoin

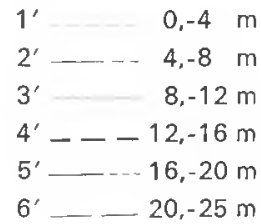

CENTRE TECHNIQUE DU GÉNIE RURAL, DES EAUX ET DES FORETTS

Division "Loisirs et Chasse "

Domaine des Barres

45290 NOGENT-SUR-VERNISSON

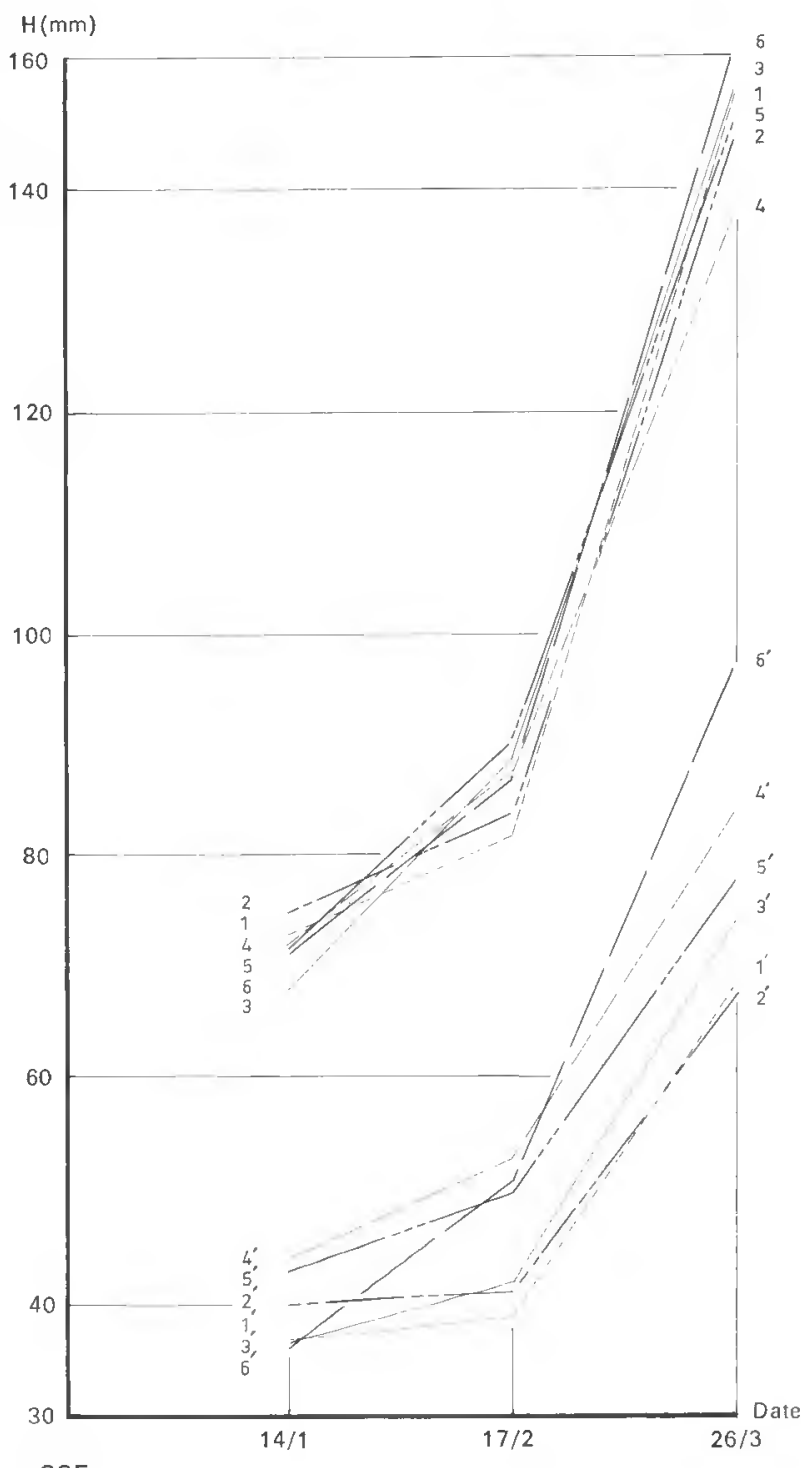




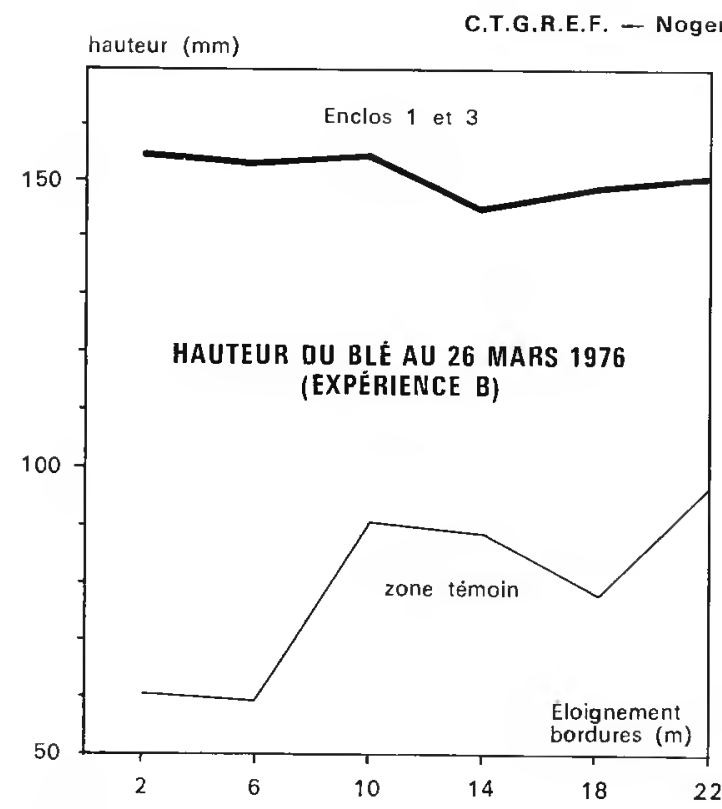

Graphique no 2
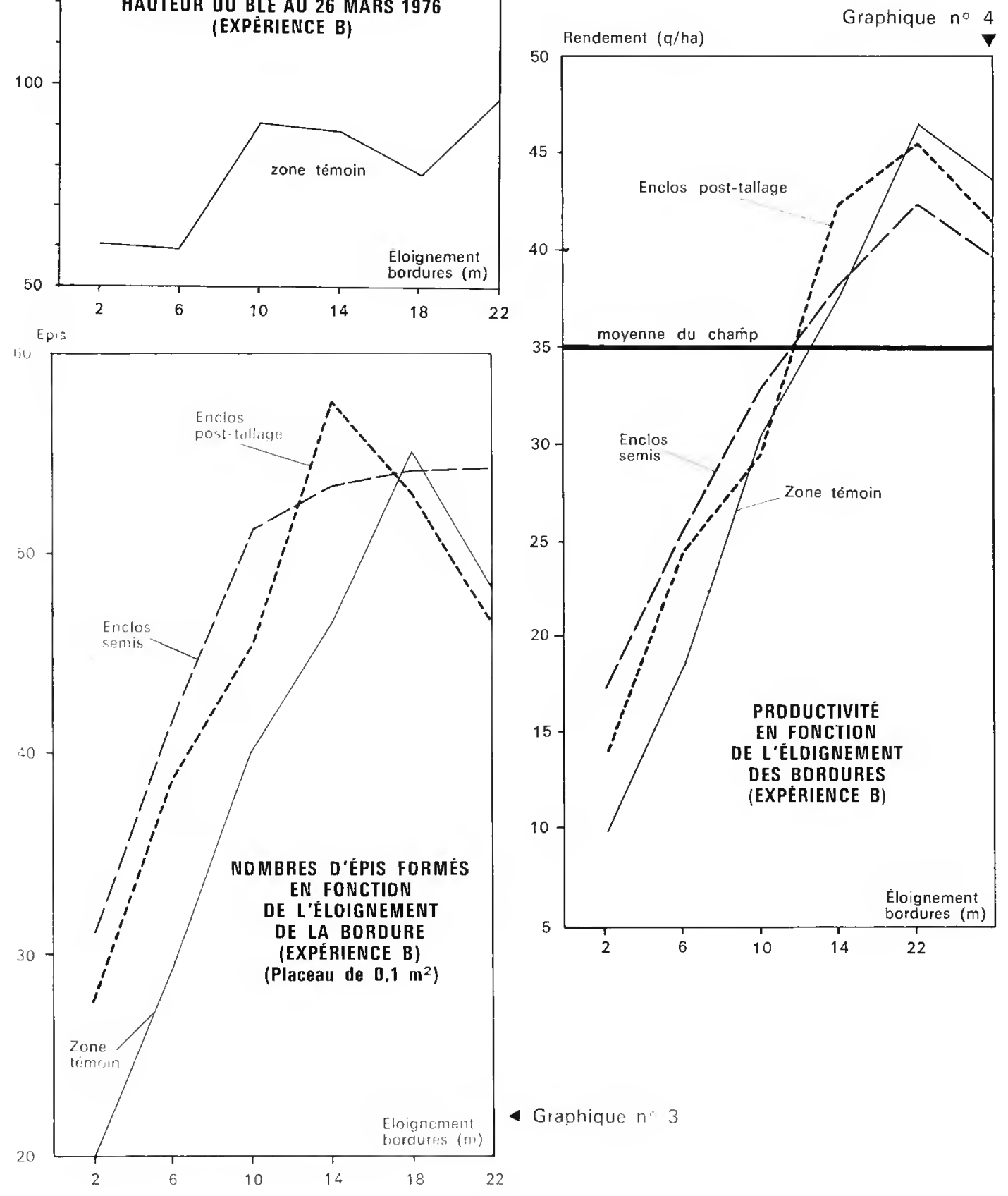